\title{
Mujeres estudiantes en Programación: entre la reproducción y las nuevas construcciones de género. El caso de la formación en el nivel medio técnico en la Ciudad de Buenos Aires ${ }^{1}$
}

\author{
Women Students in Computer Programming: Between Reproduction and \\ New Gender Constructions. The Case of Education and Training \\ at Mid-level Technical in the City of Buenos Aires
}

\author{
Claudia Jacinto, Verónica Millenaar, Eugenia Roberti, Alejandro Burgos y Mariana Sosa²
}

\section{Resumen}

El artículo busca contribuir a los debates sobre la (des)igualdad de género desde las voces de los y las estudiantes en escuelas de nivel medio técnico. Generalmente, los antecedentes de investigación se centran en la educación técnica industrial. Sin embargo, en la formación en nuevas tecnologías informáticas las desigualdades se reproducen y se resisten en una sociedad sensibilizada por cuestiones de género. ¿̇ qué se atribuye la escasa presencia de mujeres en Programación en la Ciudad de Buenos Aires? ¿Cómo se reproducen y/o cuestionan los estereotipos de género en esta formación en particular? ¿De qué modo el contexto socio-histórico, aparece influyendo sobre las representaciones y expectativas de los jóvenes? La profusa bibliografía sobre el tema señala la persistencia de las desigualdades de género en la educación y el empleo. Sin embargo, estos procesos no son inmutables, como lo muestra la amplia instalación de agendas de género en las sociedades occidentales durante las últimas décadas. Inmutabilidad, transformaciones y prácticas disruptivas coexisten y se retroalimentan.

El artículo analiza la manera en que se construyen y también se cuestionan los estereotipos de género, focalizándose en las siguientes dimensiones: a) pertinencia de la educación técnica para varones y mujeres; b) razones de elección de esta particular especialidad; c) tratos diferenciados de parte de docentes y compañeros; d) división sexual del trabajo y e) expectativas a futuro. La estrategia metodológica adoptada fue triangulación de datos cuantitativos y cualitativos, recogidos en base a una muestra de tres escuelas técnico-profesionales estratégicamente elegidas.

\section{Palabras clave}

Formación tecnológica, desigualdad de género, educación media, métodos mixtos.

\section{Abstract}

The article seeks to contribute to the debates about gender (in)equality from the voices of the technical middle level schools' students. Generally, research focuses on industrial technical education. However, inequalities are as well reproduced and resisted in technology and computer programming training, in a gender-sensitized society. Why is the presence of women in computer programming training so limited in the City of Buenos Aires? How are gender stereotypes reproduced and/or questioned in this particular education? How does the socio-historical context appear to influence the representations and expectations of young people?

The profuse bibliography on the subject indicates the persistence of gender inequalities in education and employment. However, these processes are not immutable as shown by the extensive installation of gender agendas in Western societies during the last decades. Immutability transformations and disruptive practices coexist and feed back. The article discusses how gender stereotypes are constructed but also questioned, focusing on the following dimensions: a) relevance of technical education for men and women; b) reasons for choosing this particular specialty; c) differentiated treatment by teachers and classmates; d) sexual division of labor and e) future expectations. The methodological strategy adopted was triangulation of quantitative and qualitative data, collected based on a sample of three strategically chosen technical-professional schools.

\section{Keywords}

Technological training, gender inequality, secondary education, mixed methods.

\section{Cómo citar/Citation}

Jacinto, Claudia; Millenaar, Verónica; Roberti, Eugenia; Burgos, Alejandro y Sosa, Mariana (2020). Mujeres estudiantes en Programación: entre la reproducción y las nuevas construcciones de género. El caso de la formación en el nivel medio técnico en la Ciudad de Buenos Aires. Revista de Sociología de la Educación-RASE, 13 (3), 432-450. http://dx.doi.org/10.7203/RASE.13.3.16605.

\footnotetext{
Este artículo desarrolla una parte de los hallazgos de una investigación más amplia del Programa de Estudios sobre Educación, Juventud y Trabajo (PREJET). Fue financiada por el Fondo Nacional de Investigaciones de Educación Técnico-Profesional (FoNIETP) del Instituto Nacional de Educación Tecnológica (INET) del Ministerio de Educación, Cultura, Ciencia y Tecnología.

2 Claudia Jacinto, claudiajacinto01@gmail.com; Verónica Millenaar, veronicamillenaar@gmail.com; Eugenia Roberti, eugenia.roberti@hotmail.com; Alejandro Burgos; Mariana Lucía Sosa.
} 


\section{Introducción ${ }^{3}$}

Las desigualdades de género en la educación y el empleo se construyen procesual y relacionalmente en el marco de «tramas» complejas, entre las cuales las dinámicas económicas y sociales -contextuadas geográfica, institucional y sectorialmente- ocupan un lugar clave. Esas relaciones sociales están atravesadas también por las orientaciones de las políticas educativas y laborales, las tendencias culturales e históricas (Jacinto, 2016).

Una amplia bibliografía señala que la formación de una identidad de género se construye desde una etapa muy temprana de la vida. La familia, la escuela y los medios de comunicación contribuyen a sostener, en un hacer reiterativo, estereotipos de género. Parte de los efectos diferenciados del género se observan en los intereses y/o en la elección de opciones escolares, donde las niñas tienden a alejarse de las disciplinas científicas. A su vez, dichas aspiraciones educativas se traducen en futuros compromisos profesionales, que reducen el rango de posibilidades para las mujeres (Duru Bellat, 2016).

Sin embargo, estos procesos no son inmutables como lo muestra la amplia instalación de agendas de género en las sociedades occidentales durante las últimas décadas. Inmutabilidad, transformaciones y prácticas disruptivas coexisten y se retroalimentan plenas de tensiones en cada contexto socio-histórico. En Argentina, la agenda pública de género ha experimentado una amplia movilización desde principios de siglo. Cuestiones como la identidad de género y la violencia sexual, impulsados por los movimientos de mujeres y otros grupos diversos, han logrado una significativa visibilidad mediática, amplio debate público y nuevas legislaciones ${ }^{4}$.

En este marco, el presente artículo, se enfocará sobre las permanencias y las mutaciones de las desigualdades de género en la educación técnica de nivel medio (EST); una modalidad cuya historia está atravesada por dichas segregaciones. Generalmente, la investigación en torno a la temática se centró en la educación técnica industrial pero, más recientemente, se ha observado que en la formación en nuevas tecnologías informáticas persisten sesgos de género. Para develar las formas en que éstos últimos se reproducen y se resisten en una sociedad sensibilizada por cuestiones de género, se investigó el caso de la formación en Programación en la Ciudad de Buenos Aires ${ }^{5}$. Esta especialidad tiene relevancia dentro de la matrícula del nivel (10\%), y refleja importantes brechas educativas y laborales basadas en género.

El artículo, centrándose en la perspectiva estudiantil (en diálogo con las percepciones de directores y docentes), abordará los siguientes interrogantes: ¿a qué se atribuye la escasa presencia de mujeres en la especialidad? ¿Cómo se reproducen y/o cuestionan los estereotipos de género? ¿De qué modo el particular contexto socio-histórico, aparece influyendo sobre las representaciones y expectativas de los jóvenes?

Metodológicamente, la investigación más amplia adoptó un enfoque de triangulación metodológica, que utilizó varias técnicas y fuentes de información. Para este artículo, se analizaron datos provenientes

3 Este artículo contempla las orientaciones para el empleo de un lenguaje inclusivo en cuanto al género en español castellano, elaboradas por la Organización de las Naciones Unidas, que considera tres criterios a fines de hacer un uso más justo y preciso del lenguaje: 1) evitar expresiones discriminatorias, 2) visibilizar el género cuando la situación comunicativa lo requiere y, 3) no visibilizarlo cuando no resulta necesario.

4 Más aún, si bien los temas de género tienen una historia extensa de lucha y conquistas, la «agenda de género» ha ganado la calle, principalmente, a través de la campaña «Ni una menos» en contra de los femicidios, la convocatoria al Paro Internacional de Género del 8 de marzo y la campaña por la sanción de la Ley de Interrupción Legal y Voluntaria del Embarazo.

5 La Educación Técnico-Profesional de nivel secundario en Argentina dura entre 6 y 7 años (de los 12/13 a los 17/18), compartiendo los primeros tres años con la educación secundaria general. O sea, la formación especializada comienza alrededor de los 15/16 años, resultando exigente por su doble turno y su alta carga de materias teóricas y tecnológicas. Según el marco de referencia correspondiente, el título certifica habilidades para participar en el desarrollo de software desempeñando roles que tienen por objeto producir programas, módulos o componentes de sistemas de computación. 
de una muestra de tres instituciones estratégicamente elegidas ${ }^{6}$, entre las doce, que brindaban la especialidad de Programación. Se aplicaron como técnicas de recolección de datos: 1) una encuesta basada en cuestionarios estructurados (cerrados) a 120 estudiantes del tercero y cuarto año de la especialidad. Las dimensiones abordadas en la encuesta fueron aprendizajes, expectativas de educación y trabajo, tareas realizadas en el hogar, características sociodemográficas, etc.; 2) seis grupos focales en los que participaron estudiantes del último año, que se realizaron separando varones y mujeres. Se utilizó una guía de preguntas disparadoras y actividades que giraron en torno a percepciones sobre desigualdades de trato, estereotipos y expectativas a futuro?

Las tres escuelas son públicas, y ubicadas en barrios de clase media, media-baja. Por su parte, los estudiantes provienen de sectores medios-bajos (con diferencias entre las escuelas); y de hogares con clima educativo medio (pocos son profesionales) y ninguno tiene ocupaciones vinculadas a la informática.

\section{Desigualdades de género en la Educación Técnico-Profesional: el caso de la Progra- mación}

Las investigaciones previas sobre educación secundaria técnica en Argentina muestran que la primera segregación de las mujeres se relaciona con la no «elección» de la modalidad y, en particular, de las especialidades vinculadas a estereotipos masculinos. En el caso de la Informática, se ha señalado que las diferencias en el acceso se vinculan con que la matemática, el pensamiento lógico, la hiper-competencia, el carácter menos social de la formación, etc., son características atribuidas a los varones (Morgade, 2009).

Estas percepciones estereotipadas en torno a las habilidades y a la división sexual del trabajo se encuentran también presentes en las dinámicas de las instituciones educativas. Los códigos de género ${ }^{8}$ aparecen en la cotidianeidad de las instituciones educativas y en las prácticas de enseñanza escolares. Se perciben valores, creencias y normas explicitadas o no de lo que significa ser mujer y ser varón, que legitiman ciertas interacciones y deslegitiman otras. Este proceso es reforzado por la naturalización de ciertos rasgos considerados poco menos que innatos (los varones por naturaleza son «más inteligentes», «saben más», «les resulta más fácil»). En el caso de las estudiantes, para tener éxito en la escuela deben quebrar el «orden natural» (Morgade y Kaplan, 1999). Muchas veces desconociendo el carácter arbitrario de esas disposiciones culturales las alumnas adhieren sin plena conciencia, es decir, de modo tácito o implícito, y hacen propios estos juicios.

Aún cuando el discurso institucional homogeneizador está centrado en el trato igualitario de varones y mujeres, las estudiantes manifiestan una y otra vez cierto padecimiento por su condición de género (Bloj, 2017). En muchos casos, se les prohíbe manipular máquinas o se les asignan tareas diferentes por el mero hecho de ser mujeres (León, 2009; Seoane, 2013). Muchas de ellas intentan pasar inadvertidas como mujeres tras la pretensión de ser tratadas como «iguales».

6 Las instituciones fueron seleccionadas de acuerdo a su «tasa de feminidad». La proporción de mujeres en la especialidad de Informática ronda el 14\% (UEICEE, 2016). Teniendo en cuenta esta referencia se seleccionaron: a) una institución cuya matrícula femenina en la especialidad fuera proporcionalmente similar a la observada en el total de Escuelas; b) otra cuyo porcentaje fuera significativamente menor al nivel general y finalmente; c) otra cuya tasa de feminidad sea ampliamente superior al valor del conjunto.

7 Los grupos focales se desarrollaron siguiendo el marco metodológico propio de los grupos de discusión, como técnica de recolección de datos cualitativos (Scribano, 2008). En total participaron 42 estudiantes en los grupos focales, siendo los grupos masculinos más numerosos que los femeninos (por su escasa presencia en dichas escuelas). Participaron estudiantes de los últimos años para generar discusiones basadas en una experiencia educativa de más largo plazo. En cuanto al procesamiento cualitativo, la información procedente de entrevistas se procesó a través de matrices de datos y a través de re-elaboraciones de los datos de grupos focales por medio de informes analíticos sobre la base de categorías seleccionadas y reportes de campo.

8 Subirats los define como «las formas y procesos que definen, limitan y trasmiten el conjunto de modelos socialmente disponibles para que los individuos jóvenes lleguen a una identificación personal en términos de hombre o mujer» (1986: 382). 
Ahora bien, ¿qué formas toman estos procesos en el campo específico de la Informática? Se han aportado diferentes explicaciones a la escasez de mujeres en las tecnologías, dado que es un fenómeno común a muchos países. Como en otros campos, esta desigualdad deriva de patrones patriarcales reflejados en la socialización. A pesar de que las mujeres sostienen carreras escolares más exitosas que los varones, suelen presentar desventajas en matemática (INET, 2015); se les transmite que la ciencia, la técnica y la tecnología son temas e intereses de varones y que ellas carecen de estas habilidades. Si bien la Informática no responde exactamente al prototipo tradicional de la educación técnica industrial, las desigualdades son fuertes tanto en el desarrollo de vocaciones, como en la auto-discriminación.

$\mathrm{Al}$ respecto, una encuesta representativa a estudiantes del nivel secundario en el área metropolitana de Buenos Aires (Fundación Sadovsky, 2015) mostró que la proporción de quienes proyectaban estudiar Informática en el nivel superior era de siete varones por cada mujer. Armar y desarmar objetos, aprender autónomamente, hacer tareas de matemática y lógica, estar frente a una computadora por un tiempo prolongado (habilidades asociadas a los procesos productivos de software) eran consideradas como masculinas. Entre los usos de la informática, los juegos complejos ${ }^{9}$ presentaban la asimetría más importante: los varones los elegían siete veces más que las mujeres como primera opción asociada al uso de la computadora en su vida cotidiana; en cambio, las mujeres la utilizaban en primer lugar para redes sociales. Como un dato revelador, el estudio encuentra una fuerte asociación entre la realización de juegos complejos y la elección de una carrera superior en Informática en los dos sexos.

Respecto al mercado de trabajo, la inserción de las mujeres en el sector informático responde a una constante revelada en los estudios sobre segregación laboral: cuanto más cerca está un trabajo técnico relacionado con el proceso de producción y cuanto mayor sea su valor social (en términos de su papel en la reproducción social, lo que sucede con las nuevas tecnologías), menos probable es que sea realizado por una mujer (Cereq, 1992).

La relación entre las mujeres y las tecnologías ha estado históricamente reducida a una imagen tecnofóbica, que las visibiliza como contrarias al uso de las tecnologías (González Ramos et al., 2017). Los entornos laborales en el sector tienen reglas, costumbres y códigos que pueden resultar hostiles a las mujeres, razón por la cual se detectan fenómenos similares que en las instituciones escolares: su masculinización e invisibilización.

Sin embargo, no todo es inmutabilidad: siguiendo a Butler ([1990] 2007), el género se construye performativamente, en cada práctica reiterativa, y por lo tanto, los códigos de género no pueden ser pensados como fijos e invariantes. Como sostiene Molina (2012), más allá de que los discursos escolares evoquen la esencialidad masculina y femenina atribuyéndoles rasgos fijos, la trama escolar es también un interesante escenario para la problematización de dicho sustancialismo. Es decir, a la vez que la escuela encarna y tiende a reproducir normas hegemónicas de género, constituye un espacio de disputa en torno a la producción de género y al despliegue de luchas sociales que configuran nuevas construcciones genéricas, protagonizadas por las estudiantes. En los siguientes apartados, se discutirán hallazgos de la investigación acerca de las persistencias y variaciones en los discursos y prácticas estudiantiles, de la mano de la configuración de una nueva agenda de género.

9 Los «juegos complejos», por oposición a la categoría de «juegos de Facebook y parecidos», requieren de la comprensión y asimilación de un conjunto de reglas más desarrolladas, que implican habilidades de concentración, organización, configuración, análisis, etc. sobre las cuales se podrían erigir habilidades informáticas ulteriores (Fundación Sadovsky, 2015). 


\section{Segregaciones en el mercado de trabajo de la Ciudad de Buenos Aires y agenda de género: ¿dos cuestiones independientes?}

La Ciudad de Buenos Aires encabeza el ranking nacional de acuerdo al Índice de Desarrollo Sostenible Provincial de Argentina, presentando una distancia considerable respecto a las demás provincias, al contar con los niveles más elevados de crecimiento económico, inclusión social y sostenibilidad ambiental (PNUD, 2017).

A pesar que el acceso y terminación de la educación (incluso al nivel universitario) favorece a las mujeres, en el terreno laboral se manifiestan las mayores desigualdades entre ambos géneros. Si bien en el contexto nacional, la brecha laboral entre varones y mujeres resulta muy significativa, en la Ciudad presenta menores diferencias, aunque éstas pueden identificarse al observar indicadores básicos tales como: tasa de desocupacion (mujeres $9 \%$, varones $7 \%$ ), inactividad (mujeres $39 \%$, varones $22 \%$ ), informalidad (mujeres $26 \%$, varones $23 \%$ ), promedio de horas trabajadas por semana (mujeres 42 , varones 50 ), subocupación demandante (mujeres $18 \%$, varones $17 \%$ ), etc. ${ }^{10}$

La diferencia en la tasa de inactividad puede vincularse a la realización de tareas de cuidado en el hogar. Cuando hay hijos menores de 18 años en el hogar, la brecha por género en la actividad aumenta, ya que los varones con hijos incrementan su participación mientras que la de las mujeres madres disminuye.

La rama de la Informática constituye un sector dinámico, con demanda creciente y convocante para los jóvenes, pero las mujeres representan solo el $28 \%$ de los ocupados (Sosa, 2018) ${ }^{11}$. Esta desigualdad de inserción en el sector es llamativa, teniendo en cuenta la demanda insatisfecha de trabajadores, las elevadas tasas de rotación y la competencia entre firmas por la contratación de los mismos empleados; que se refleja en salarios superiores, en promedio a los trabajadores de otros sectores, y muchos beneficios apreciados por los jóvenes ${ }^{12}$.

Pese a la relevancia que tienen las brechas de género en el mercado de trabajo, el tema se encuentra relativamente invisibilizado en el debate público. Aunque la normativa argentina asume muchos compromisos destinados a mejorar la situación de las mujeres en el mundo laboral, las políticas al respecto son limitadas: presentan escasa cobertura y fragmentación de las intervenciones, como asimetría en las capacidades institucionales de las entidades subnacionales para ejecutarlas. A su vez, la implementación de una oferta pública de cuidado y desarrollo infantil avanza muy lentamente (Díaz Langou et al., 2019).

En cambio, otros temas atravesaron la agenda de género y tuvieron particular impacto en las escuelas, uno de más sustantivos fue la sanción de la Ley 26150 de Educación Sexual Integral (ESI) en 2006 (Faur, 2018). Esta ley enfatiza una aproximación integral y positiva a la sexualidad, incluyendo temáticas asociadas a las relaciones afectivas, género, diversidad y derechos, entre otros objetivos de aprendizaje. En la Ciudad de Buenos Aires, si bien su implementación se demoró, se sancionó una ley jurisdiccional en 2010 que ha orientado las políticas públicas en la materia ${ }^{13}$.

10 Fuente: procesamientos propios en base a INDEC-EPH,2t, 2017.

11 En la Ciudad de Buenos Aires, el sector servicios informáticos representa un 2,9\% del PBI local. Incluso, se constata que el $70 \%$ de la industria TIC a nivel nacional se encuentra radicada en la Ciudad (sobresaliendo el rubro el desarrollo de software, que emplea a más del 50\% de los ocupados en la actividad) (OPSSI,2016).

12 Casi la totalidad de los ocupados $(90 \%)$ tiene nivel secundario completo o más, seis de cada diez se desempeña en puestos de calificación elevada y el $81 \%$ son asalariados, la mayoría formales (Sosa, 2018).

13 Cabe señalar que, en parte a raíz de la movilización de los temas de género, nuevas iniciativas fueron llevadas adelante. Así, el INET crea en el 2018 la Comisión de Género de la ETP, cuyo objetivo es promover acciones destinadas a incorporar la perspectiva de género de forma transversal, en toda la educación técnico-profesional. De algún modo, el cambio cultural y las nuevas resistencias a los mandatos de género, comienzan a ser tomadas por las políticas de la modalidad, al menos incipientemente. 
Particularmente, las discriminaciones también alcanzan el acceso a la formación en Programación a nivel medio en la Ciudad. Aunque se trata de una de las especialidades con mayor matrícula (10\% del total de EST) la distribución por género es desigual. Mientras que en el total de la modalidad técnica, la matrícula femenina ronda el 25\%, en Informática representa el 14\% (UEICEE, 2016). ¿Cuáles son las razones de esta escasa participación? ¿Perciben los jóvenes los obstáculos respecto al acceso, tratos diferenciados y expectativas futuras segmentadas genéricamente? En los próximos puntos, se analizarán los estereotipos y sesgos de género que enfrentan, reproducen y resisten las estudiantes de carreras de Programación.

4. Articulaciones y disputas en la trama cotidiana escolar: de la «invisibilidad» a la resistencia cultural femenina

\section{1. La experiencia escolar en clave de género: entre la histórica invisibilización y las «nuevas chicas»}

Como ha corroborado una vasta evidencia empírica, la cultura escolar es parte de una construcción histórica que fue configurando relaciones de discriminación en género: los libros de textos escolares con contenido sexista, la relación del profesorado con sus alumnas, el material didáctico, los atributos físicos destacados como ideales, etc. Todos estos elementos constituyen un currículum oculto que históricamente ha reproducido roles y visiones discriminatorias, pero que, actualmente, presenta ciertas resistencias.

En la investigación desarrollada, estas resistencias y cuestionamientos hicieron evidentes las muchas aristas de las desigualdades de género que se expresan a lo largo de toda la experiencia escolar, de diversas maneras. De acuerdo a los datos de la encuesta, las mujeres resultaron más críticas respecto a las percepciones de discriminación en el proceso pedagógico, evidenciando una búsqueda por explicitar un lugar de desventaja. Ellas tienden más que los varones a observar prácticas discriminatorias por género en los talleres y laboratorios, así como en relación al acceso a prácticas y pasantías. Como se observa en la Tabla I, la diferencia del acuerdo de las mujeres respecto a si ellas son tratadas del mismo modo que sus compañeros en los espacios de taller y laboratorios, es de más de 20 puntos $^{14}$. La única coincidencia, respecto a que no habría desigualdades de trato, concierne a la exigencia impartida por los docentes.

Tabla I. Percepciones en torno al trato diferenciado en el proceso pedagógico, por sexo

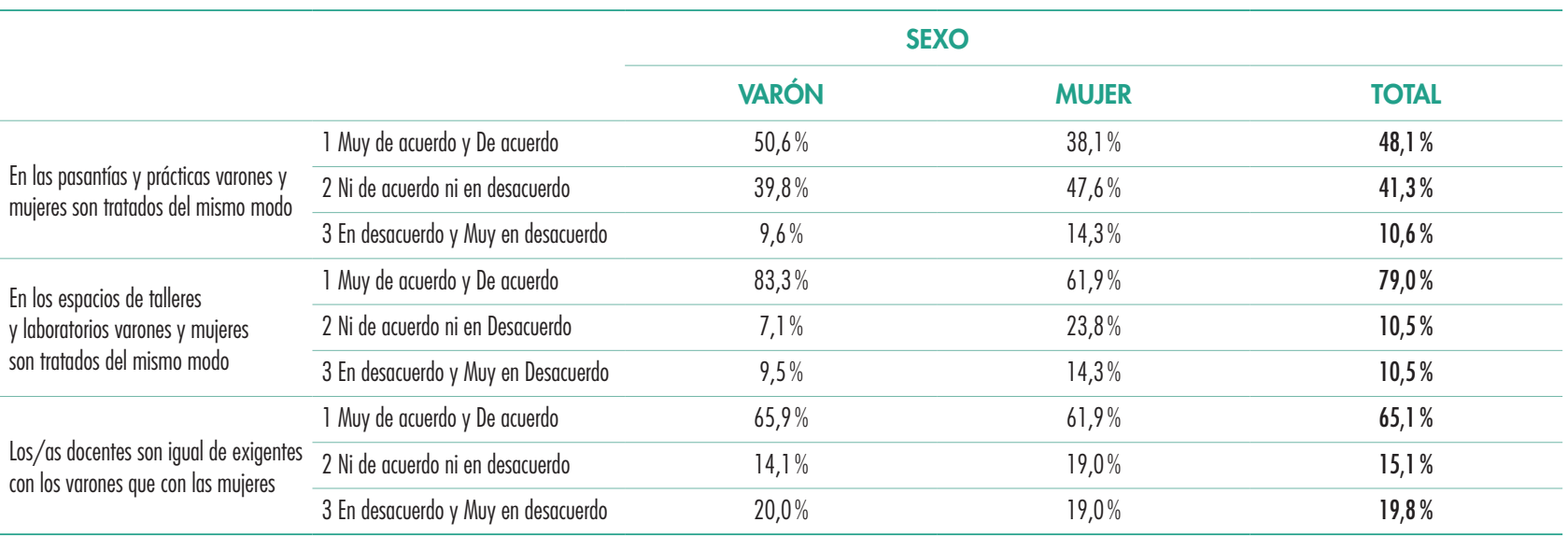

Fuente: elaboración propia en base a datos del Proyecto INET «Construyendo la igualdad de género en la educación técnico-profesional. Estudio de casos en CABA».

14 El taller consiste en un espacio de formación práctica vinculada a diferentes oficios que se realiza en forma rotativa en contra-turno durante los dos primeros años de la escuela técnica. A partir de tercer año se concentra en el laboratorio de informática. 
Tal como se refleja en la Tabla I, en los grupos focales también los estudiantes ponen de manifiesto la persistencia de prácticas que sostienen la diferenciación de género en su experiencia escolar. Aunque aún se observa una invisibilización de dichas desigualdades, también aparecen voces femeninas que señalan críticamente el trato cotidiano de sus compañeros y docentes. Los relatos reflejan tanto cuidados «especiales» hacia las mujeres (que las terminan ubicando en una posición de desventaja) como su exclusión de las tareas prácticas.

Los reclamos de las estudiantes parecen expresar la intolerancia a formas de discriminación anacrónicas y otrora naturalizadas, que están comenzando a ser cuestionadas por las «nuevas alumnas». Así, frente a la histórica asimilación de la educación técnica como una alternativa solo para varones, se percibe una mayor apertura y crecimiento de la proporción de mujeres en sus escuelas: «hoy está aumentando el número de chicas» (Grupo focal mujeres. Escuela C). Las estudiantes perciben una mayor presencia de matrícula femenina, cuestionando el sesgo de género arraigado en la modalidad:

\section{"Generalmente la escuela técnica está vista para hombres. Ahora no tanto, ahora sí se animan las mijeres». (Grupo focal mujeres. Escuela C).}

En los grupos focales surge la idea de cómo las chicas han cambiado. También en las entrevistas, directivos y docentes expresan que se están produciendo algunos corrimientos respecto a los estereotipos. Los adultos entrevistados perciben la presencia de un cambio cultural en cuanto a las formas de ser mujer y al lugar en que las jóvenes se posicionan. En efecto, repetidamente sostienen que las mujeres «cambiaron», en el sentido de que las chicas actualmente aparecen con otra actitud, «incluso más desenvueltas» que los varones -claro está, que esto ha sido en un largo proceso-. Estas percepciones se vinculan no solo a la experiencia escolar sino también al contexto general en que la agenda pública de género aparece en diferentes esferas de la cotidianeidad.

En el mismo sentido, en los grupos focales se observa el valor positivo que le otorgan a la formación práctica del taller, reemplazando un «código de género» tradicionalmente androcéntrico, que las mujeres logran romper al concurrir a una escuela técnica:

«Me sacó el miedo de agarrar un serrucho o una lima, antes pensaba "no voy a poder"; me enseñó que no es así, que si tenés ganas de hacerlo podés, no importa si sos mujer u hombre. Y también cómo desenvolverte en la vida en realidad. Me llevó cicatrices, porque me corté, pero perdí el miedo». (Grupo focal mujeres. Escuela C).

Los relatos de las estudiantes visibilizan que adquirir y asimilar conocimientos que son culturalmente reservados para los varones, tiene una incidencia positiva en sus subjetividades, en el afianzamiento y determinación de una imagen de sí mismas que incide en la manera de pensar, actuar e interpretar la realidad, así como de relacionarse con los pares.

"Como mujer, me llevo mucho orgullo de acá, porque te forman para ser líder, que no haya diferencias entre varones y mujeres, y está genial porque ningún profesor nunca biz̧o ninguna diferencia. Yo soy la única mujer en el curso y nunca tuve problemas. Me voy muy contenta en ese sentido, sé hacerme valer». (Grupo focal mujeres. Escuela C). 
Si recuperamos las voces femeninas se observan inicios de ruptura con los estereotipos de género; ellas con sus prácticas sociales y culturales construyen una «subcultura escolar de resistencia» a la cultura tradicional y androcéntrica imperante en las instituciones educativas (McLaren, 2005 [1984]). Las estudiantes se convierten en un «sujeto de resistencia» que se evidencia en la ruptura de los códigos culturales y sociales que se les asignaban como mujeres. Sin embargo, la construcción de una «subcultura escolar de resistencia» no es un proceso lineal y encuentra obstáculos entre las propias mujeres que lleva a que se auto-atribuyan la responsabilidad por su escasa participación en carreras o estudios considerados típicamente masculinos.

Para recapitular, podemos decir entonces que los testimonios de las estudiantes permiten vislumbrar señales del proceso de deconstrucción de estereotipos de género asociados a la escuela técnica como un espacio escolar típicamente masculino. El material empírico evidencia avances, tensiones y contradicciones en el intento de promover una cultura escolar inclusiva e igualitaria entre varones y mujeres. Sin embargo, como hemos mencionado, se perciben en los discursos la convivencia de prácticas escolares diferenciadoras con otras más cuestionadoras y rupturistas. Cabe recordar que las mujeres entrevistadas son minoría en todas las escuelas estudiadas. De allí que, quienes llegan a finalizar el ciclo superior podrían encuadrarse en la tradicional figura de «mujeres fuertes» que logran superar los obstáculos diversos a presentes a lo largo de toda la experiencia escolar.

\subsection{Mujeres programadoras: la elección de la especialidad}

La decisión en torno a la elección de la escuela está vinculada a múltiples razones y atravesada por condicionamientos de todo tipo, según muestra la bibliografía. La medida en que estas elecciones son decisiones individuales o familiares racionales según la teoría del costo-beneficio ha sido muy discutida en el medio de los márgenes contextuales, socio-económicos y culturales en que se producen estas elecciones. El tipo y calidad de la oferta educativa, las distancias y costos del desplazamiento, la información accesible y el capital cultural y social de las familias parecen grandes condicionantes, así como las propias trayectorias escolares previas y/o las propensiones y/o habilidades mostradas en ellas. Entre los factores que influyen en esas decisiones, los estereotipos de género juegan especialmente, y lo hacen con particularidad en la elección de una especialidad técnica, como es la Programación.

Hace unos años, el Censo Nacional de Estudiantes Técnicos de 2009 mostró que, en relación a la elección de la escuela técnica, a los varones los motiva «el gusto por la tecnología» más que a las mujeres; sin embargo, en ambos prevalecían razones vinculadas a mejorar las expectativas de inserción laboral posterior y/o continuar estudios universitarios. El grupo de jóvenes encuestados en nuestra investigación muestra motivaciones similares: dentro de los motivos que lleva a los estudiantes a elegir una escuela técnica se destaca la posibilidad de conseguir un trabajo en el futuro, y esto se observa especialmente en el caso de las mujeres.

Ambos sexos eligieron la escuela por la especialidad. Un dato interesante es que, según la encuesta (Tabla II), para los varones el motivo que adquiere mayor peso es «porque me gusta» (6 de cada 10); en cambio, entre las mujeres es «porque creo que me va a ayudar a conseguir trabajo» (5 de cada 10). Como se advertía en el estudio de 2009, en los varones el gusto por la tecnología aparece en su vida cotidiana como un motor de su interés por la especialidad. Esa mayor motivación, lejos de ser espontánea, está enraizada en los procesos de socialización primaria. En las mujeres, parece primar una 
motivación más pragmática, avizorando un futuro laboral mejor. Como desarrollamos en la siguiente sección, esta afirmación debe analizarse respecto a sus posibilidades de inserción laboral, no necesariamente circunscriptas a la especialidad de la informática.

Tabla II. Motivaciones para elegir la especialización en Informática, por sexo

\begin{tabular}{|c|c|c|c|c|}
\hline & & \multicolumn{3}{|c|}{ SEXO } \\
\hline & & VARÓN & MUJER & TOTAL \\
\hline \multirow{4}{*}{$\begin{array}{l}\text { ¿Cuál es el principal motivo por el cual elegiste } \\
\text { ESTA ESPECIALIZACIÓN en particular? }\end{array}$} & Porque me gusta & $64,0 \%$ & $28,6 \%$ & $57,0 \%$ \\
\hline & Porque creo que me va a ayudar a conseguir trabajo & $17,4 \%$ & $47,6 \%$ & $23,4 \%$ \\
\hline & Porque me prepara para la carrera terciaria/universitaria que quiero seguir & $16,3 \%$ & $14,3 \%$ & $15,9 \%$ \\
\hline & Otros motivos & $2,4 \%$ & $9,6 \%$ & $0,9 \%$ \\
\hline
\end{tabular}

Fuente: elaboración propia en base a datos del Proyecto INET «Construyendo la igualdad de género en la educación técnico-profesional. Estudio de casos en CABA».

De este modo, las relaciones previas con los dispositivos informáticos parecen configurar la vocación para seleccionar esta especialidad. En consonancia con lo que señala la literatura, respecto a la brecha de género existente en la alfabetización digital, el entretenimiento a través de los videojuegos complejos aparece como una práctica androcéntrica y con poca llegada a las mujeres (Rubio Méndez, 2012). Tal como surge en los grupos focales, las propias estudiantes atribuyen una «falta de interés» del sexo femenino hacia la especialidad, lo cual explicaría su escasa presencia en las escuelas:

«yo creo que los chicos están más metidos en el tema; me parece que a ellos les gusta más». (Grupo focal mujeres. Escuela B).

«Todo pasa por el interés y no por el género. Si las chicas tienen interés tendrían las mismas posibilidades». (Grupo focal mujeres. Escuela C).

También son las mismas mujeres, no obstante, las que consideran que el menor rendimiento de sus compañeros se vincula a la falta de motivación por el aprendizaje escolar, manifestándose ellas con mayor confianza en sus procesos de aprendizaje, incluso en posición de minoría dentro de la técnica.

«Solo piensan en terminar el día, llegar a su casa y sentarse en la computadora a jugar. Yo ya lo pasé, es como que no tenés aspiraciones. Yo veo a mis compañeros: se sacan un 1 o cosas así, y a veces no les importa. O se llevan mal con algún profesor y tampoco les importa». (Grupo focal mujeres. Escuela A).

De este modo, las mujeres que eligieron la especialidad, expresan conocer con mayor detalle en qué consiste, que los propios varones, e incluso manifiestan una mayor conciencia respecto a su exigente formación curricular teórico-práctica, como muestra el siguiente relato:

«Principalmente, a los nenes de 14 para abajo les aclaro que si van a seguir esta especialidad no van a hacer videojuegos, ni se la van a pasar jugando. Que si quieren les explico acerca de lo que van a hacer, van a programan». (Grupo focal mujeres. Escuela C). 
En efecto, las mujeres, menos habituadas a la práctica de los videojuegos y menos aproximadas al mundo de la informática como entretenimiento, evidencian una mayor necesidad de información sobre la especialidad. Así, ya en la misma elección de la especialidad se manifiestan elementos de género que operan en los deseos, intereses y necesidades ${ }^{15}$.

Los estereotipos de género también aparecen al interior del hogar, incidiendo en los apoyos u obstáculos que brinda la familia a la hora de definir la elección de la especialidad. En el caso que sigue, la disyuntiva contrasta una especialidad considerada «femenina», como Administración, con la Programación. La estudiante en cuestión, que decidió por gusto personal y expectativa a futuro estudiar Programación, se encontró con que esa elección fue rechazada en su ámbito familiar:

"Yo entré más a ciegas, pero porque era lo que más me gustaba. Mi mamá estaba en contra de
que siguiera esta carrera, quería que siguiera Administración de empresas, pero me revelé [...].
Cuando yo estaba por decidir, no se veía muy fuerte esta carrera. Si vos elegías Administración
de empresas, ibas a trabajar en un banco, tener un sueldo seguro y una vida pasable. Ella quería
eso para mí, y yo quería seguir lo que a mi me gustara». (Grupo focal mujeres. Escuela A).

El relato muestra cómo algunas estudiantes van logrando quebrar el estereotipo de género sobre ciertas carreras más «adecuadas» para ellas, incluso con el costo de verse en posición minoritaria en dichas especialidades. No obstante, para enfrentar dicho estereotipo es preciso mostrar una fuerte determinación, que vislumbra procesos de resistencia femenina. Esto es observado en estudios que abordan los cambios culturales en la ETP tradicional, a partir de la mayor autonomía en la elección de las especialidades (Evans, 2006).

No hay duda de que en la escuela técnica persisten expectativas sociales y culturales vinculadas al género, donde se espera que sean las mujeres quienes acaten las normas de la cultura escolar. Para McLaren (2005 [1984]) en la escuela uno incorpora -no sin resistencias- los roles o actitudes esperados a los sexos. Así, la cultura escolar transmite los valores, normas y pautas que regulan los comportamientos adecuados a cada una de estas categorías. Esta transmisión se concreta en la institución y principalmente en la sala de clase, donde los docentes reproducen el sistema jerárquico de divisiones y clasificaciones de género, que no cuestionan ni problematizan, sino que refuerzan a pesar de que en su discurso teórico se promueva la igualdad en género.

En esta investigación enfocada en Programación, la totalidad de los docentes entrevistados declaró no hacer diferencias de género en su práctica de enseñanza. Sostuvieron que al no tratarse de una especialidad «sucia» o que implica esfuerzo físico, la participación resulta igualitaria por género. En palabras de un docente: «en nuestra especialidad se trabaja en el laboratorio de Informática. Las tareas se distribuyen a todos por igual), invisibilizando los estereotipos de género que persisten aún en la elección de la carrera. En última instancia, al mismo tiempo que se vislumbran resistencias y cuestionamientos por parte de las estudiantes, persisten también prácticas que abonan al sostenimiento de la desigualdad de género y su escasa problematización.

15 De cualquier manera, la práctica de videojuegos complejos sólo facilita, de ningún modo determina, una vocación posterior por continuar Informática en la educación superior (Fundación Sadosky, 2015). 


\section{Mérito o sesgo de género: percepciones sobre división sexual del trabajo productivo y reproductivo y expectativas a futuro}

Una temática arduamente debatida por la literatura radica en la percepción de desigualdades de género en el mercado de trabajo, sin embargo, ¿qué peculiaridades presentaría la inserción laboral en Programación desde las voces de las y los estudiantes? Para responder estos interrogantes, a continuación analizaremos la (in)visibilización de las diferencias de género que se oculta tras la perspectiva de igualdad de oportunidades; prestando especial atención tanto a los procesos de reproducción de las discriminaciones genéricas como también a las resistencias, miradas críticas y estrategias que desarrollan las mujeres entrevistadas.

Si bien las y los estudiantes entrevistados no tienen aún experiencia en el mercado de trabajo, en sus percepciones a futuro suelen considerar que la trayectoria laboral dependería más del «mérito» ${ }^{16}$, que de otras diferenciaciones: «toman al que sabe más», «la contratación tiene que ver con el conocimiento de cada uno». Detrás de esta creencia en la meritocracia se legitiman posiciones desiguales en el mercado laboral, las cuales son asignadas en función de las capacidades y esfuerzos individuales, desconociéndose e invisibilizándose la condición de género.

Precisamente, en los grupos focales, tanto mujeres como varones estudiantes mencionaron que no esperarían experimentar diferencias o discriminación por género a la hora de ingresar en el mercado de trabajo, ya sea en términos generales o en relación a la especialidad de Programación: «no es una cuestión de género, es cuestión de personalidad», «es el carácter de la persona, no importa si es hombre o mujen» (Grupo focal mujeres. Escuela C). En sus percepciones las capacidades cognitivas y las habilidades socio-emocionales serían posibles elementos que a futuro incidirían en el ingreso al mercado de trabajo, independiente de la cuestión de género.

\section{"Yo creo que siendo mujer tengo la misma posibilidad que un hombre. Ahora, si yo no tengo tantos conocimientos que un hombre, asumo que van a contratar a un hombre». (Grupo focal mujeres. Escuela B).}

En complementación con estas percepciones, la encuesta muestra que son ellas quienes más valoran la formación en competencias personales o socio-emocionales, más allá de las habilidades técnicas: con una discrepancia de más de 20 puntos, las estudiantes señalan que les gustaría que la escuela las prepare en competencias ligadas a la responsabilidad, autoestima, sociabilidad y autocontrol (mujeres 42,9\%, varones $22,1 \%$ ). A pesar de estas diferencias, en las expectativas de formación a futuro, tanto varones como mujeres, señalan la importancia de tener conocimientos en computación e idiomas y de contar con el título técnico como requisitos principales.

Resulta interesante señalar que las percepciones y expectativas de los actores institucionales entrevistados coinciden con la de los estudiantes. Docentes y directivos se manifiestan optimistas sobre las posibilidades de inserción laboral de los egresados, no visualizando diferencias por género, según dicen, «todo depende». Desde su perspectiva, los saberes movilizados en las transiciones laborales iniciales tienen más que ver con el denominado «saber sen»: las habilidades de comunicación (intercambio de información,

16 De acuerdo con Busso y Pérez (2019), el término meritocracia aparece por primera vez en el ensayo The rise of meritocracy $1870-2033$ del inglés Michael Young (1958). En una obra póstuma, Weber, ya señalaba que «el que está mejor situado siente la urgente necesidad de considerar como 'legítima' su posición privilegiada, de considerar su propia situación como resultado de un 'mérito'» (1969 [1922]: 705). Desde allí el discurso meritocrático sostiene que cada uno recibe, alcanza o posee lo que merece, según el esfuerzo o virtud individual. Desde las ciencias sociales, una vasta literatura ha cuestionado el carácter meritocrático de la sociedad contemporánea (Reygadas, 2004; Dubet, 2012). 
fomento de relaciones interpersonales) y las actitudes frente a la tarea, que con el saber propiamente técnico. En última instancia, los «merecedores» de las mejores posiciones laborales son aquellos jóvenes que demuestran cualidades personales ligadas con la pro actividad, la disposición al trabajo y el cumplimiento de reglas. En este sentido, en las percepciones de los docentes surge también de manera recurrente la afirmación del mérito como forma de selección diferencial a la hora de ingresar al mercado de trabajo, invisibilizándose el modo en que los desempeños laborales se encuentran atravesados por el género.

Sin embargo, la situación del mercado de trabajo está lejos de responder a una lógica meritocrática. Incluso, la mayor demanda de educación por parte de las mujeres resulta una estrategia que busca revertir indicadores laborales desiguales (Martínez García, 2019). Pese a que numerosos estudios demuestran la persistencia de segregaciones de género ligadas al mundo del trabajo, desde las voces de los entrevistados las mismas se desdibujan otorgando centralidad a las competencias técnicas adquiridas - «lo que se sabe»-, dato que contrasta con la desigual forma de valorizar el título que brinda la especialidad (Tabla III). Allí, el diploma presenta un valor desigual, a igual titulación mujeres y varones tienen proyecciones de insertarse diferencialmente en el mercado de trabajo, alcanzando ellos mejores condiciones laborales en términos de vinculación con la carrera estudiada.

\section{Tabla III. Percepción sobre la relación del futuro empleo con la especialidad estudiada, por sexo}

\begin{tabular}{lcc}
\hline & \multicolumn{2}{c}{ SEXO } \\
\cline { 2 - 3 } 1.En un trabajo directamente relacionado con la especialidad técnica & VARÓN & MUJER \\
\hline 2.En otro trabajo en el que aplique algunos conocimientos aprendidos en la especialidad técnica & $36,5 \%$ & $25,0 \%$ \\
\hline 3.En cualquier otro trabajo para un egresado de nivel medio & $47,6 \%$ & $55,0 \%$ \\
\hline
\end{tabular}

Fuente: elaboración propia en base a datos del Proyecto INET «Construyendo la igualdad de género en la educación técnico-profesional. Estudio de casos en CABA».

La desigualdad de género se observa en que varones y mujeres vislumbran percepciones diferenciales respecto a sus oportunidades laborales futuras. Más de la mitad de los varones cree que al finalizar el nivel medio podrá acceder a un trabajo directamente relacionado con la Programación, mientras que en el caso de las mujeres solo el $25 \%$ lo considera una posibilidad. De este modo, ellas perciben que deberán enfrentar prácticas de selección discriminatorias en el sector; sin embargo, son optimistas al sostener que podrán utilizar sus conocimientos en informática en otros sectores de actividad.

En este punto, resulta interesante contrastar la escasa problematización de las discriminaciones en el mercado de trabajo sectorial, que lleva a no visualizar que la informática es un sector masculinizado. El discurso «meritocrático» de que dicha especialidad, por sus características de trabajo poco sucio y de poco esfuerzo físico, «no tiene género»-siendo igualmente apto tanto para varones como para mujeres-, se confronta con las afirmaciones de estudios recientes: ellas eligen menos las opciones tecnológicas para insertarse laboralmente dadas las condiciones de trabajo que caracterizan al sector, al representar un obstáculo en la conciliación con la vida familiar (Martínez García, 2019).

$\mathrm{Al}$ igual que sus docentes, los estudiantes asocian la especialidad que cursan como una de las razones por las que no se evidenciarían marcadamente discriminaciones de género en el mercado laboral. En este sentido, se diferencian de lo que sucedería con los egresados de carreras técnicas industriales: las mujeres 
podrían experimentar discriminaciones en ocupaciones donde el imperativo para realizar la actividad sea la fuerza, como en tareas relacionadas con la construcción. Los relatos resultan significativos:

\begin{abstract}
"Apostaría más a un varón en trabajos de fuerza [...]. Creo que siendo una mujer y trabajando en una obra le va a costar más. Yo creo que voy a elegir a un hombre porque se necesita más fuerza o físico». (Grupo focal mujeres. Escuela C).
\end{abstract}

«Para mí la diferencia de género con lo único que tiene que ver es con lo físico». (Grupo focal varones. Escuela B).

Sin embargo, el estudio de González Ramos et al., 2017 realizado en España acerca de la participación laboral de las mujeres en el sector tecnología, contradice estas posturas. Señala que una causa del escaso porcentaje en la participación femenina es la creciente masculinización de los entornos laborales. Similar a lo que ocurre en la escuela, allí se propiciaría un clima de trabajo poco «amigable» para las mujeres, interpeladas a entablar una competencia desigual con códigos de intercambio masculino. En consecuencia, buscan desarrollar estrategias de «supervivencia» para sostenerse en estos entornos laborales: tratan de pasar desapercibidas, para no ser vistas como posibles competidoras de los varones, o se ven «obligadas» a adecuar sus conductas y emociones al modelo de socialización cultural androcéntrico.

Un ejemplo ilustrativo de la persistencia de discriminaciones laborales en la especialidad opera en las formas tradicionales de gestión del personal, diferenciadas por género, que implementan las empresas: «está el estereotipo que al hombre lo toman mucho más que a la mujens (Grupo focal mujeres. Escuela B). Ahora bien, aunque los relatos dejan traslucir la continuidad de prácticas «machistas» de selección, las y los estudiantes las consideran propias de una etapa de la sociedad que se estaría superando, en sus propias palabras:

"Igual, varones o mujeres, como lo ven a uno, le dan la entrevista, creo que no tendrían problemas. Salvo que el tipo sea un machista, es igual. Hoy estamos más tranquilos con esto». (Grupo focal varones. Escuela $C)$.

"A mi no me tocó vivir nada de eso [discriminación por género], pero estoy segura que debe haber. Todavia sigue el machismo, por el lado de que se piensa que las computadoras no son para las mujeres y que es trabajo de hombres». (Grupo focal mujeres. Escuela A).

Resulta llamativo que, son las mujeres, quienes deben sortear los filtros selectivos. Así, es ilustrativo que, desde la visión de los estudiantes entrevistados, cuando se presentan prácticas discriminatorias en los empleadores, se asume como una responsabilidad individual la superación de ese obstáculo. Frente a lo que se evidencia una condición adscripta -en términos de género-, se busca una solución individual en base a cualidades personales: aquellas que desarrollan un «carácter fuerte» logran imponerse en la selección para un puesto de trabajo. Un estudiante varón decía sobre una compañera del mismo curso:

«Depende de cada uno, porque si uno quiere seguir adelante, va a ir seguir buscando sin parar. $Y$ depende de la experiencia. Ella tiene una cabeza que nos gana a todos. Y tiene su manera de discutir. Pelea mucho, defiende sus ideas». (Grupo focal varones. Escuela A). 
Un hallazgo de la investigación es que las mujeres resultan más críticas respecto a las percepciones de discriminación en el mercado de trabajo, evidenciando una búsqueda por resistir y explicitar un lugar de desventaja. De acuerdo a los datos de la encuesta, si bien más de la mitad de los casos tienen opiniones que pueden ubicarse dentro de una percepción no estereotipada de género, los varones presentan mayor tendencia a sostener ciertos estereotipos (Tabla IV). Por ejemplo, ellos tienden a pensar con mayor frecuencia que ciertos trabajos son solo para varones o solo para mujeres. También, cuestionan menos la idea de que son mejores para desempeñarse como jefes, mientras que ellas se manifiestan muy en desacuerdo con esta afirmación. Incluso, reflejan concepciones más estereotipadas respecto a las posibilidades de desempeño de sus coetáneas en ocupaciones vinculadas a la Informática; en particular, los varones se perciben mejor preparados para realizar la actividad de «reparación de computadoras»: mientras un $24,4 \%$ de ellos cree que habrá diferencias de desempeño entre los sexos, en las mujeres desciende a un $5 \%$.

Como ya ha mencionado la evidencia investigativa, si a eso sumamos las desventajas femeninas en el cuidado de hermanos, niños y responsabilidades del hogar -que afectan en mayor proporción a mujeres que a varones- entonces las desventajas se multiplican y complejizan. Más enfáticamente, los estereotipos prevalecen en cuestiones ligadas a la distribución de tareas del hogar: mientras el 95\% de las mujeres manifiesta son actividades que no competen solamente a ellas, en los varones ese acuerdo solo llega al $67 \%$ (Tabla IV).

Tabla IV. Percepciones sobre el trabajo (dentro y fuera del hogar) de varones y mujeres, por sexo

\begin{tabular}{|c|c|c|c|c|}
\hline & \multicolumn{4}{|c|}{ SEXO } \\
\hline & & VARÓN & MUJER & TOTAL \\
\hline \multirow{3}{*}{$\begin{array}{l}\text { ¿Las tareas del hogar son principalmente } \\
\text { responsabilidad de las mujeres? }\end{array}$} & 1 Muy de acuerdo y De acuerdo & $11,6 \%$ & $0,0 \%$ & $9,3 \%$ \\
\hline & 2 Ni de acuerdo ni en desacuerdo & $20,9 \%$ & $4,8 \%$ & $17,8 \%$ \\
\hline & 3 En desacuerdo y Muy en desacuerdo & $67,4 \%$ & $95,2 \%$ & $72,9 \%$ \\
\hline \multirow{3}{*}{ ¿Algunos trabajos deben ser solo para varones? } & 1 Muy de acuerdo y De acuerdo & $20,9 \%$ & $4,8 \%$ & $17,8 \%$ \\
\hline & 2 Ni de acuerdo ni en Desacuerdo & $30,2 \%$ & $38,1 \%$ & $31,8 \%$ \\
\hline & 3 En desacuerdo y Muy en Desacuerdo & $48,8 \%$ & $57,1 \%$ & $50,5 \%$ \\
\hline \multirow{3}{*}{ ¿Algunos trabajos deben ser solo para mujeres? } & 1 Muy de acuerdo y De acuerdo & $16,7 \%$ & $0,0 \%$ & $13,3 \%$ \\
\hline & 2 Ni de acuerdo ni en desacuerdo & $32,1 \%$ & $38,1 \%$ & $33,3 \%$ \\
\hline & 3 En desacuerdo y Muy en desacuerdo & $51,2 \%$ & $61,9 \%$ & $53,3 \%$ \\
\hline \multirow{3}{*}{$\begin{array}{l}\text { ¿Los varones son mejores que las mujeres para } \\
\text { desempeñarse como jefe? }\end{array}$} & 1 Muy de acuerdo y De acuerdo & $12,8 \%$ & $9,5 \%$ & $12,1 \%$ \\
\hline & 2 Ni de acuerdo ni en desacuerdo & $30,2 \%$ & $14,3 \%$ & $27,1 \%$ \\
\hline & 3 En desacuerdo y Muy en desacuerdo & $57,0 \%$ & $76,2 \%$ & $60,7 \%$ \\
\hline
\end{tabular}

Fuente: elaboración propia en base a datos del Proyecto INET «Construyendo la igualdad de género en la educación técnico-profesional. Estudio de casos en CABA».

De acuerdo a los datos producidos en base a los grupos focales, el discurso «meritocrático» se comienza a complejizar y resquebrajar cuando los relatos recabados dejan traslucir la persistencia de ciertos estereotipos que reproducen desigualdades de género: «las chicas tienen las mismas posibilidades que los varones, salvo el tema de ser mamás» (Grupo focal varones. Escuela C). Este último testimonio da cuenta que la asunción de responsabilidades reproductivas es considerada una fuente mayúscula de segregación laboral para las mujeres y, por tanto, son uno de los principales determinantes de las brechas de género en el mercado de trabajo (Díaz Langou et. al., 2019). 
Sintetizando, en las percepciones y expectativas de los estudiantes de Programación respecto a la futura inserción laboral se evidencian viejas inequidades adscriptas, que presentan al mismo tiempo nuevas luces y sombras: si, por una parte, se invisibilizan diferenciaciones de género en el futuro laboral, por otra parte, aparecen diferencias: a) acerca del alcance de los estereotipos, siendo las mujeres las que menos acuerdan con ellos; y b) también son ellas quienes presentan mayor tendencia a visibilizar obstáculos en el sector informático, que se suman a las mayores responsabilidades asumidas en las tareas de cuidado y el trabajo no remunerado.

\section{A modo de conclusión}

El artículo se centra en la construcción y el cuestionamiento de las desigualdades de género, en el marco de la formación en escuelas técnicas de nivel medio. En general, la bibliografía muestra el carácter sexista de la escuela técnica industrial. Esta investigación se orienta específicamente a la especialidad de Programación, en la cual también se evidencian estas desigualdades, en el marco de una sociedad sensibilizada por cuestiones de género. Si bien la Informática no se asocia con las especialidades tradicionales de la escuela técnica, allí las desigualdades también son evidentes. El acceso a la tecnología y su uso en la vida cotidiana, ya muestra una socialización de género que luego incide en las expectativas y experiencias diferenciadas en el marco de la educación técnica. No obstante, el artículo vislumbra también procesos de cambio y formas de resistencias desarrolladas principalmente por las estudiantes.

En cuanto a la experiencia durante la escolaridad, las mujeres que logran llegar al ciclo orientado de la EST de Programación, perciben un trato igualitario, aunque con ambigüedades y cuestionamientos, al resultarles ostensibles las diferencias con sus compañeros respecto a los espacios de talleres y otras actividades de aplicación. A estas diferenciaciones, se suma la continuidad de imágenes estereotipadas de género respecto a las cualidades atribuidas a varones y mujeres, en particular, las capacidades lógico-matemáticas de unos y otras.

Si, por un lado, las estudiantes en algunos casos naturalizan y se pliegan a dichos juicios, no visualizando discriminaciones; por otro lado, ellas resultan más críticas que los varones respecto a las desigualdades y/o a la calidad de los aprendizajes, presentando una fuerte valoración subjetiva sobre los saberes que adquieren en la educación técnica, tanto para la vida cotidiana como también para la futura inserción educativa y/o laboral.

Se evidencia las relaciones de poder que estructuran el aula y la socialización escolar, y sus consecuencias en las experiencias de las estudiantes que resultan subalternizadas, prestando especial atención a cómo las estudiantes cuestionan y resisten a estas lógicas discriminatorias de género. En este sentido, el estudio permite comprender que hay un vínculo -y a veces retroalimentación- entre desigualdades sociales, culturales y laborales, el género y las resistencias.

De allí que, una decisión desde las políticas de buscar revertir las discriminaciones en la educación técnica, solo a través de la mayor presencia de mujeres en las aulas de dicha modalidad, es un camino insuficiente; en tanto las desigualdades también se hacen presentes a lo largo de toda la cursada, obstaculizando el proceso de aprendizaje, las confianzas de las alumnas mujeres y su sostenimiento en las instituciones.

Más específicamente, perdura asimismo una segregación entre varones y mujeres respecto a la especialidad de Programación. El papel de las identificaciones de género en la elección de la especialidad, pone 
de relieve que los sentidos otorgados a las mismas reproducen un estereotipo sexista. Las construcciones ideológicas de larga data no facilitan las «vocaciones» en dicha especialidad, que adquiere un carácter masculinizado desde el imaginario colectivo y el mercado de trabajo; a su vez, la persistencia de brechas de alfabetización digital entre ambos géneros, incide en el alejamiento de las mujeres de la Informática.

Sobre todo, un terreno donde ellas observan que tendrán menores oportunidades es en la futura inserción laboral en el sector, pese a contar con las mismas credenciales que sus pares varones. Esta percepción se confirma a la luz de los datos secundarios recabados sobre el sector, donde se vislumbra que ellas encuentran menos posibilidades de utilización de sus saberes técnicos. Asimismo, se verifica que entre las mujeres ocupadas en la rama existe una mayor proporción de tituladas universitarias que entre los varones, lo cual estaría mostrando una mayor exigencia hacia las mujeres en términos de credenciales dentro del sector ${ }^{17}$. La segregación horizontal resulta así el techo que operará en los primeros tramos de los procesos de inserción de las mujeres programadoras. Aunque el mercado de trabajo es menos igualador para las mujeres, no siempre se cuestiona el sesgo de género oculto tras el velo de la meritocracia.

\section{Referencias bibliográficas}

Bloj, Cristina (2017). Trayectorias de mujeres Educación técnico-profesional y trabajo en la Argentina. Santiago de Chile: Naciones Unidas/CEPAL (en línea). https://www.cepal.org/es/publicaciones/41230-trayectorias-mujeres-educacion-tecnico-profesional-trabajo-la-argentina, consultado el 1 de diciembre de 2019.

Busso, Mariana y Pérez, Pablo (2019): "El velo meritocrático: inequidades en la inserción laboral de jóvenes durante el gobierno de Cambiemos". RevIISE Revista de Ciencias Sociales y Humanas, 13 (13), 133-145.

Butler, Judith ([1990] 2007). El género en disputa. El feminismo y la subversión de la identidad. Buenos Aires: Paidós.

CENUAETP (2009). Censo Nacional de Último Año de Educación Técnico Profesional. Buenos Aires: INET.

CEREQ (1992): "Women in 'Male' Careers: The Case of Higher Technicians in France, Training and Employment, French Dimensions". CEREQ, (6).

Díaz Langou, Gala et. al., (2019). El género del trabajo: entre la casa, el sueldo y los derechos. Buenos Aires: CIPPEC (en línea). https://www.cippec.org/wp-content/uploads/2019/11/el_genero_del_trabajo.pdf, consultado el 10 de diciembre de 2019.

Dubet, François (2012). Repensar la justicia social. Contra el mito de la igualdad de oportunidades. Buenos Aires: Siglo XXI.

Duru Bellat, Marie (2016). “Ã l'école du genre”. Enfances et Psy, (69), 90-100.

Evans, Karen (2006): “Achieving Equity Through 'Gender Autonomy': The Challenges for VET Policy and Practice". Journal of Vocational Education \& Training, 58 (4), 393-408.

Faur, Eleonor (2018). El derecho a la educación sexual integral en la Argentina. Aprendizajes de una experiencia exitosa. Ministerio de Educación de la Nación, Argentina: Organización de Naciones Unidas.

17 Porcentaje de mujeres con nivel universitario completo ocupadas en rama TIC: $46 \%$. Porcentaje de varones con nivel universitario completo ocupados en rama TIC: $35 \%$ Fuente: INDEC-EPH-2018, 3t. 
Fundación Sadosky (2013). Y las mujeres... ¿Dónde están? Primer estudio de la Fundación Dr. Manuel Sadosky sobre la baja presencia femenina en informática. Buenos Aires: Fundación Sadosky, Ministerio de Ciencia, Tecnología e Innovación Productiva (en línea). http://www.fundacionsadosky.org.ar/wp-content/ uploads/2015/05/resumen-mujeres-y-computacion-2013.pdf, consultado el 6 de septiembre de 2019.

González Ramos, Ana et. al., (2017): "Las mujeres en el mercado de trabajo de las tecnologías". Revista Española de Investigaciones Sociológicas, 159, 73-90. (en línea). DOI: http://dx.doi.org/10.5477/cis/ reis.159.73, consultado el 14 de agosto de 2019.

INET (2015). La Educación Técnico-Profesional en Cifras. Buenos Aires: Ministerio de Educación-Instituto Nacional de Educación Tecnológica. http://www.inet.edu.ar/wp-content/uploads/2013/03/CifrasETP_Argentina2015_ampliado.pdf, consultado el 4 de enero de 2019.

Jacinto, Claudia (Coord.) (2016). Protección social y formación para el trabajo de jóvenes en la Argentina reciente. Entramados, alcances y tensiones. Buenos Aires, Argentina: (en línea). http://ides.org.ar/wp-content/ uploads/2016/11/Libros-del-IDES-2016-PREJET-Jacinto.pdf, consultado el 9 de mayo de 2020.

León, Fernanda (2009): "Mujeres y discurso pedagógico en la escuela técnica" en Alícia Villa (comp.): Sexualidad, relaciones de género y de generación, Perspectivas histórico-culturales en educación. Buenos Aires: NOVEDUC.

Martínez García, José Saturnino (2019). Género y educación: brecha inversa y segregación. Equidad, educación y género. Madrid: Editorial Dykinson.

McLaren, Peter ([1984] 2005). La vida en las escuelas. Una introducción a la pedagogía critica en los fundamentos de la educación. Buenos Aires: Siglo XXI.

Molina, Guadalupe (2012): “Construcciones de género en la escuela secundaria”. Cuadernos de Educación, $\mathrm{X},(10), 1-15$.

Morgade, Graciela (2009): "Educación, relaciones de género y sexualidad: caminos recorridos, nudos resistentes" en Alícia Villa (comp.): Sexualidad, relaciones de género y de generación, Perspectivas histórico-culturales en educación. Buenos Aires: NOVEDUC.

Morgade, Graciela y Kaplan, Carina (1999): "Mujeres esmeradas y varones inteligentes: Juicios escolares desde un enfoque de género”. Revista Argentina de Educación. Buenos Aires: AGCE.

Observatorio Permanente de la Industria de Software y Servicios Informáticos (OPSSI) (2016). Reporte anual del sector de software y servicios informáticos de la República Argentina.

PNUD (2017). Informe Nacional sobre Desarrollo Humano 2017 del PNUD Argentina Información para el desarroIlo sostenible. Argentina y la Agenda 2030.

Reygadas, Luis (2004): "Las redes de la desigualdad: un enfoque multidimensional”. Política y Cultura, (22), $7-25$.

Rubio Méndez, María (2012): "Videojuegos y género: propuestas para una reapropiación eficaz del medio". Comunicación presentada en el IX Congreso Iberoamericano de Ciencia, Tecnología y Género. Sevilla.

Scribano, Adrián (2008). El proceso de investigación social cualitativo. Buenos Aires: Prometeo. 
Seoane, Viviana (2013). Géneros, cuerpos y sexualidades. Experiencias de mujeres en escuelas técnicas de la ciudad de La Plata (Tesis de doctorado). Universidad de Buenos Aires, Argentina.

Sosa, Mariana (2018): "Inserción laboral de egresados técnicos en dos sectores contrastantes: construcción e informática" en Claudia Jacinto (coord.): El secundario vale. Saberes, certificados y títulos técnicos en la inserción laboral de jóvenes. Buenos Aires: Miño y Dávila.

Subirats, Marina (1986): "Niños y niñas en la escuela: una exploración de los códigos de género actuales". en Mariano Enguita (ed.): Marxismo y sociología de la educación. Madrid: Akal.

UEICEE (2017). Relevamiento Anual. Investigación y Estadística. Unidad de Evaluación Integral de la Calidad y Equidad Educativa, Secretaria de Educación, CABA.

Weber, Max ([1922] 1964). Economía y sociedad esbozo de sociología comprensiva. México: FCE.

\section{Notas biográficas}

Claudia Jacinto es Socióloga, UBA. Doctora en Estudios de América Latina-Sociología, Université París III. Investigadora Principal del Consejo Nacional de Investigaciones Científicas y Técnicas de Argentina, con sede en el CIS-IDES. Coordinadora del Programa de Estudios Juventud, Educación y Trabajo desde 2007. Ex-coordinadora de la Junta de Calificación y Promoción del CONICET. Miembro de la Academia Internacional de Educación. Docente de posgrado en la UBA y Flacso.

Verónica Millenaar es Socióloga (UBA), Magíster en Ciencias Sociales (UNGS) y Doctora en Ciencias Sociales (UBA). Es investigadora asistente en CONICET, con sede de trabajo en el Programa de Estudios sobre Juventud, Educación y Trabajo (PREJET- CIS- IDES/CONICET). Es además Jefa de Trabajos Prácticos en la Facultad de Ciencias Sociales de la UBA. Es miembro de la Comisión Directiva de la Asociación de Especialistas en Estudios del Trabajo (ASET). Sus investigaciones han abordado las relaciones entre educación y trabajo, las trayectorias educativo-laborales de jóvenes desde una perspectiva de género, los saberes y la formación para el trabajo y las masculinidades y feminidades en la Formación Profesional (FP).

ORCID: https://orcid.org/0000-0001-8084-4745.

Eugenia Roberti es Licenciada en Sociología, Magíster en Ciencias Sociales y Doctora en Ciencias Sociales por la Universidad Nacional de La Plata (UNLP, Argentina). Actualmente se desempeña como Investigadora Asistente del Consejo Nacional de Investigaciones Científicas y Técnicas (CONICET), son sede en la Universidad Nacional de Arturo Jauretche (UNAJ, Argentina). También, es docente de grado y de posgrado en diversas universidad nacionales de dicho país. Miembro del Programa de Estudios sobre Juventud, Educación y Trabajo (PREJET-IDES/CIS). Sus principales temáticas de interés son los jóvenes, los estudios biográficos, la desigualdad social, las políticas públicas y las relaciones educación-formación-trabajo. Forma parte de diversos proyectos de investigación y ha elaborado numerosas publicaciones en revistas nacionales e internacionales en relación a estas temáticas. Es autora del libro 
«Prácticas laborales y fragmentación social. La difícil transición de la escuela al trabajo», Noveduc/Aulas y Andamios, 2016.

Alejandro Burgos es Licenciado en Educación por la Universidad Nacional de General Sarmiento. Magíster en Educación: pedagogías críticas y problemáticas socioeducativas por la Facultad de Filosofía y Letras de la Universidad de Buenos Aires. Doctor en Ciencias Sociales por la Facultad de Ciencias Sociales de la Universidad de Buenos Aires. Becario posdoctoral en el Consejo Nacional de Investigaciones Científicas y Técnicas con sede en el Centro de Investigación Social-Instituto de Desarrollo Económico y Social. Docente en Institutos de Formación Docente y Técnica. Integrante del Programa de Estudios en Educación, Juventud y Trabajo dirigido por Claudia Jacinto en temas de sociología de la educación, pedagogía, educación de jóvenes y adultos, formación para el trabajo, trayectorias educativas, desigualdad educativa.

Mariana Lucía Sosa es Socióloga (UBA), Magíster en Sociología Económica (IDAES-UNSAM) y Doctora en Ciencias Sociales (UBA). Becaria postdoctoral en CONICET, con sede de trabajo en el Programa de Estudios sobre Juventud, Educación y Trabajo (PREJET- CIS-IDES/CONICET). Temas de investigación: transiciones educación-trabajo, inserción laboral y educativa, movilidad intergeneracional de egresados de educación técnico-profesional en distintas ramas de actividad económica. Abordaje cuantitativo a partir de la generación, procesamiento y análisis de datos estadísticos. Utilización de técnicas de análisis multidimensional, tales como construcción de tipologías, análisis de componentes principales (ACP), análisis Factorial de Correspondencias Múltiples (ACM), análisis de clasificación (ACL), regresiones, etc. 